\title{
Fractional Israel layers
}

\author{
J. P. Krisch ${ }^{\text {a) }}$ \\ Department of Physics, University of Michigan, Ann Arbor, Michigan 48109
}

(Received 5 June 2006; accepted 14 October 2006; published online 22 December 2006)

\begin{abstract}
A fractional Lie derivative, valid in the thin shell limit, is developed. The nonlocal nature of the fractional derivative allows the inclusion of shell thickness in the stress energy description of zero thickness Israel layers. The method is applied to several examples. (C) 2006 American Institute of Physics.
\end{abstract}

[DOI: $10.1063 / 1.2390660]$

\section{INTRODUCTION}

Derivatives of fractional order, first described in 1695 by Leibnitz, ${ }^{1,2}$ have been successful in modeling transport processes with anomalous microscopic time and/or spatial structures. ${ }^{3-6}$ Among the transport applications are diffusion equations fractional in both time and spatial coordinates $^{7-9}$ and fractional Schrödinger equations; ${ }^{10,11}$ there are applications in biophysics and thermodynamics ${ }^{12}$ as well as texts ${ }^{13,14}$ on solving the fractional differential equations arising in transport. Other areas of application are the inclusion of dissipative processes in a Lagrangian formalism by introducing fractional derivatives as generalized coordinates ${ }^{15,16}$ and the use of a fractional derivative in the metric match over a boundary layer ${ }^{17,18}$ in general relativity.

The field equations of general relativity are not easily fractionally generalized because of the covariance requirement on derivatives. The general relativity (GR) applications in Refs. 17 and 18 did not modify the field equations or any of the usual GR tensors in any way; the fractional match simply provided a broader set of metric relations across a boundary. It was used to create a family of Israel layers parametrized by the noninteger order of the fractional derivative. There are, however, geometric objects used in general relativity, which can be fractionalized without altering the basic covariant structure of the theory. One of these is the Lie derivative, defined only with partial derivatives. The Lie derivatives take into account the difference between a tensor that is Taylor transported to a point and coordinate transformed at the same point. It is a local derivative. Fractional derivatives are intrinsically nonlocal, involving an integral over some region of space time. A fractional Lie derivative, while it also evaluates functional differences at a point, since it is nonlocal in its definition, could be a useful way of including nonlocal effects in single layer calculations. For example, the Israel formalism calculates the stress energy content of a single layer in terms of the jump in extrinsic curvatures across a boundary. Using a fractional Lie derivative to define a fractional extrinsic curvature would allow the inclusion of shell thickness in the standard Israel formalism. In the next section we develop a fractional Lie derivative valid to first order in a thin shell thickness. The formalism is used to describe the stress energy content of some simple fractional layers. A brief list of notation is included in the Appendix.

\section{LIE DERIVATIVE}

\section{A. The integer Lie derivative}

The Lie derivative compares the value of a function under transport from $x$ to $x^{\prime}$ and under a coordinate transform from a system of coordinates $(x)$ to a primed system $\left(x^{\prime}\right)$. The difference $V^{i}\left(x^{\prime}\right)-V^{\prime i}\left(x^{\prime}\right)$ defines the Lie derivative. Consider a vector $V^{i}$ at a point $x$. Under a coordinate transform, $x^{i \prime}=x^{i}+\xi^{i} d \lambda$, its value transforms as

\footnotetext{
${ }^{a)}$ Electronic mail: jkrisch@umich.edu
} 


$$
V^{\prime i}\left(x^{\prime}\right)=\frac{\partial x^{\prime i}}{\partial x^{a}} V^{a}(x)=\left(\delta_{a}^{i}+\frac{\partial \xi^{i}}{\partial x^{a}} d \lambda\right) V^{a}(x),
$$

where $\xi^{i}$ is the tangent to the transport path. The vector is assumed to be analytic and we have

$$
V^{i}\left(x^{\prime}\right)=V^{i}(x)+\left(\frac{\partial V^{i}}{\partial x^{\prime a}}\right)_{x}\left(x^{\prime a}-x^{a}\right)+\cdots
$$

Using Eq. (1), the Lie derivative to first order in $\xi^{i}$ is

$$
\begin{gathered}
\left(\mathcal{L}_{\xi} V^{i}\right)=\lim _{d \lambda \rightarrow 0} \frac{V^{i}\left(x^{\prime}\right)-V^{i \prime}\left(x^{\prime}\right)}{d \lambda}, \\
\left(\mathcal{L}_{\xi} V^{i}\right)=\left(\frac{\partial V^{i}}{\partial x^{\prime a}}\right)_{x} \xi^{a}-\left(\frac{\partial \xi^{i}}{\partial x^{\prime a}}\right)_{x} V^{a}(x) .
\end{gathered}
$$

Similar expressions exist for covariant vectors and tensors of higher order. For a scalar function, $F$, the Lie derivative is a simple directional derivative.

$$
\left(\mathcal{L}_{\xi} F\right)=\xi^{a}\left(\frac{\partial F}{\partial x^{\prime a}}\right)_{x}
$$

\section{B. Fractional generalization}

\section{Fractional derivative}

There are several choices to make in developing a fractional Lie derivative. The first is to choose the type of fractional derivative from among the many available. We will use the Caputo form of the fractional derivative. The Caputo fractional derivative integrates the derivative of the function while other forms take the derivative of the function integral. Among the many fractional derivative operators, the Caputo form is the closest generalization of the classical derivative. ${ }^{13} \mathrm{We}$ generalize the usual form by using partial derivatives. With $q$ as the fractional parameter, the Caputo derivative is defined for $0<q \leqslant 1$ as

$$
D_{x}^{q} V^{i}\left(x^{\prime}\right)=\frac{\partial^{q} V^{i}\left(x^{\prime}\right)}{\partial\left(x^{\prime}-x\right)^{q}}=\frac{1}{\Gamma(1-q)} \int_{x}^{x^{\prime}} d y \frac{\partial V^{i}(y)}{\partial y}\left(x^{\prime}-y\right)^{-q} .
$$

The derivative can be extended to values of $q>1$; this is discussed in the Appendix. The indices, $(q, x)$, on $D_{x}^{q}$ are not tensor indices. $q$ is the fractional parameter and $x$ is the lower limit in the integral definition. The choice of limits depends on the application being made. Our development will be for applications to thin layers of thickness $\Delta$. The coordinates near the shell are $\left(t, \eta, x^{3}, x^{4}\right)$. The spatial coordinates in the layer are $\left(x^{3}, x^{4}\right)$. The radial coordinate, $\eta$, is measured to the outer shell boundary, $\eta=R_{o}$. The integration will run from the bottom of the layer, $x=\eta$ $-\Delta$, to the top, $x^{\prime}=\eta$, with $\eta=R_{o}$ when the outer boundary size is set. With these choices, the fractional derivative definition that will be used is

$$
D_{\eta-\Delta}^{q} V^{i}(\eta)=\frac{1}{\Gamma(1-q)} \int_{\eta-\Delta}^{\eta} d y \frac{\partial V^{i}(y)}{\partial y}(\eta-y)^{-q} .
$$

The other parts of the fractional generalization to consider are the tensor transformation rule and the first terms of a fractional expansion of the tensor. These parts depend on the fractional replacement for the partial derivative. 


\section{Partial derivative and fractional derivative}

A relation between a partial derivative and a fractional derivative can be obtained by integrating Eq. (3) by parts. We have

$$
D_{x}^{q} V^{i}\left(x^{\prime}\right)=\frac{\left(x^{\prime}-x\right)^{1-q}}{\Gamma(2-q)}\left(\frac{\partial V^{i}(y)}{\partial y}\right)_{x}+\frac{\left(x^{\prime}-x\right)^{2-q}}{\Gamma(3-q)}\left(\frac{\partial^{2} V^{i}(y)}{\partial y^{2}}\right)_{x}+\cdots
$$

In the limit, $x^{\prime}-x \ll 1(\Delta \ll 1)$, the first term will dominate and we have the relation between the fractional derivative and the partial derivative to lowest order in shell thickness.

$$
\frac{(\Delta)^{1-q}}{\Gamma(2-q)}\left(\frac{\partial V^{i}(y)}{\partial y}\right)_{\eta-\Delta} \rightarrow D_{\eta-\Delta}^{q} V^{i}(\eta)
$$

For convenience, we define a fractional operator $\bigoplus_{x}^{q}$,

$$
D_{x}^{q}()=\frac{\left(x^{\prime}-x\right)^{1-q}}{\Gamma(2-q)} \bigoplus_{x}^{q}() .
$$

In the $q=1$ limit, the usual derivative is obtained. The $\bigoplus_{x}^{q}$ derivative used over a nonthin shell region, for some functions, can divide out much of the usual fractional behavior and will be used to provide the direct replacement for the partial derivative for layer examples.

\section{The first order fractional expansion}

For analytic $V^{i}$, the regular Taylor expansion is

$$
V^{i}\left(x^{\prime}\right)=V^{i}(x)+\left(\frac{\partial V^{i}(y)}{\partial y}\right)_{x}\left(x^{\prime}-x\right)+\cdots
$$

Using the fractional replacement the expansion can be written to first order as

$$
V^{i}\left(x^{\prime}\right)=V^{i}(x)+\bigoplus_{x}^{q}\left(V^{i}\right)\left(x^{\prime}-x\right)+\cdots
$$

Note that this is not a replacement for the regular Taylor expansion, complete to all orders. It will be used only to first order in the shell thickness. A complete fractional expansion was developed by Taylor and Riemann. ${ }^{19}$ Their expansion, including both fractional integrals and derivatives, is difficult to implement. Kolwankar ${ }^{20}$ have also developed a local fractional expansion.

\section{A notation addition}

The general fractional derivative

$$
D_{x}^{q} V^{i}\left(x^{\prime}\right)=\frac{\partial^{q} V^{i}\left(x^{\prime}\right)}{\partial\left(x^{\prime}-x\right)^{q}}=\frac{1}{\Gamma(1-q)} \int_{x}^{x^{\prime}} d y \frac{\partial V^{i}(y)}{\partial y}\left(x^{\prime}-y\right)^{-q}
$$

is not written with tensor functions in mind. For example, if $V^{i}\left(x^{\prime}\right)=x^{\prime i}$ we have

$$
D_{x}^{q} x^{\prime i}=\frac{\partial^{q} x^{\prime}}{\partial\left(x^{\prime}-x\right)^{q}}=\frac{1}{\Gamma(1-q)} \int_{x}^{x^{\prime}} d y \frac{\partial y^{i}}{\partial y}\left(x^{\prime}-y\right)^{-q},
$$

where $i$ has the usual coordinate index range $(1,2,3,4)$ but the coordinate identification of $x^{\prime}$ and its integral counterpart, $y$, is not clear. To clarify this an index $k$ is added to the fractional derivative notation, allowing the partial derivative to be written in index notation

$$
D_{x, k^{\prime}}^{q} x^{\prime i}=\frac{\partial^{q} x^{\prime i}}{\partial\left(x^{\prime k}-x^{k}\right)^{q}}=\frac{1}{\Gamma(1-q)} \int_{x}^{x^{\prime}} d y \frac{\partial y^{i}}{\partial y^{k}}\left(x^{\prime}-y\right)^{-q} .
$$


A similar notation is used by Samko et al. ${ }^{2}$ With this notation and using the incomplete beta function, the integral in Eq. (10) is

$$
D_{x, k}^{q} x^{\prime i}=\frac{\partial^{q} x^{\prime i}}{\partial\left(x^{\prime k}-x^{k}\right)^{q}}=\frac{\delta_{k}^{i}}{\Gamma(1-q)} \int_{x}^{x^{\prime}} d y\left(x^{\prime}-y\right)^{-q}=\frac{\delta_{k}^{i}\left(x^{\prime}-x\right)^{1-q}}{\Gamma(2-q)} .
$$

\section{The coordinate transformation}

The remaining step is to consider the transport as a coordinate transformation. The usual tensor transformation rule can be written as

$$
V^{i}(x)=\frac{\partial\left(x^{i}-x_{o}^{i}\right)}{\partial\left(x^{\prime k}-x_{o}^{\prime k}\right)} V^{\prime k}\left(x^{\prime}\right),
$$

where $x_{o}^{i}$ and $x_{o}^{\prime i}$ are the initial points in the two systems. The partial derivative in the transformation rule generalizes to

$$
\frac{\partial\left(x^{i}\right)}{\partial\left(x^{\prime k}-x^{k}\right)} \rightarrow \bigoplus_{x, k}^{q}\left(x^{i}\right)
$$

and we have

$$
V^{i}(x)=D_{x, k}^{q}\left(x^{i}\right) V^{\prime k}\left(x^{\prime}\right)=\frac{\Gamma(2-q)}{\left(x^{\prime}-x\right)^{1-q}} \frac{\partial^{q}\left(x^{i}\right)}{\partial\left(x^{\prime k}-x^{k}\right)^{q}} V^{\prime k}\left(x^{\prime}\right) .
$$

Substituting from the coordinate transformation one obtains

$$
V^{i}(x)=\frac{\Gamma(2-q)}{\left(x^{\prime}-x\right)^{1-q}}\left\{\frac{\partial^{q}\left(x^{\prime i}\right)}{\partial\left(x^{\prime k}-x^{k}\right)^{q}}-\frac{\partial^{q}\left(\xi^{i} d \lambda\right)}{\partial\left(x^{\prime k}-x^{k}\right)^{q}}\right\} V^{\prime k}\left(x^{\prime}\right) .
$$

The first integral is evaluated above giving

$$
V^{i}(x)=V^{\prime i}\left(x^{\prime}\right)-\bigoplus_{x, k}^{q}\left(\xi^{i}\right) d \lambda V^{\prime k}\left(x^{\prime}\right) .
$$

For the layer application this becomes

$$
V^{i}(\eta-\Delta)=V^{\prime i}(\eta)-\bigoplus_{\eta-\Delta, k}^{q}\left(\xi^{i}\right) d \lambda V^{\prime k}(\eta)
$$

A fractional coordinate transformation has been considered by Cotrill-Shepherd and Naber. ${ }^{21}$ Their transformation takes a fractional coordinate differential $d^{q} x^{i}$ to another $d^{q} y^{i}$ in $n$ dimensions. For $q \leqslant 1$ their transformation can be written as

$$
d x^{q, k}=\sum_{i=1}^{n} d y^{q, i} \frac{1}{\Gamma(1+q)} \frac{1}{\prod_{p=1 p \neq k}^{n}\left(x^{p}-x_{o}^{p}\right)^{q-1}} \frac{\partial\left\{\prod_{j=1 j \neq k}^{n}\left(x^{j}-x_{o}^{j}\right)^{q-1}\left(x^{k}-x_{o}^{k}\right)^{q}\right\}}{\partial\left(y^{i}-x_{o}^{\prime i}\right)^{q}},
$$

where $x_{o}^{i}$ and $x_{o}^{\prime i}$ are the initial points for the coordinate systems. In addition to the obvious term differences, this transformation is not an approximation but an exact transformation for a fractional differential defined on a fractional tangent space. The transformation used to develop the fractional Lie derivative in this paper is the fractional derivative of a regular function; it is not complete but only considers the first order terms in the shell thickness. 


\section{The fractional Lie derivative}

Using Eqs. (8) and (16) and equating $V^{i}(x)$ we have

$$
V^{i}\left(x^{\prime}\right)-\bigoplus_{x, k}^{q}\left(V^{i}\right)\left(x^{\prime k}-x^{k}\right)=V^{\prime i}\left(x^{\prime}\right)-\bigoplus_{x, k}^{q}\left(\xi^{i}\right) d \lambda V^{\prime k}\left(x^{\prime}\right) .
$$

The Lie derivative comes from the difference $V^{i}\left(x^{\prime}\right)-V^{i^{\prime}}\left(x^{\prime}\right)$. Forming the difference we have

$$
V^{i}\left(x^{\prime}\right)-V^{\prime i}\left(x^{\prime}\right)=d \lambda\left\{\bigoplus_{x, k}^{q}\left(V^{i}\right) \xi^{k}-\bigoplus_{x, k}^{q}\left(\xi^{i}\right) V^{k}\right\}
$$

The fractional Lie derivative ${ }^{q} \mathcal{L}_{\xi}\left(V^{i}\right)$ is

$$
{ }^{q} \mathcal{L}_{\xi} V^{i}=\bigoplus_{x, k}^{q}\left(V^{i}\right) \xi^{k}-\bigoplus_{x, k}^{q}\left(\xi^{i}\right) V^{k} .
$$

Similar development results in the fractional Lie derivative forms for covariant vectors and higher order tensors. The fractional Lie derivative of $V_{i}$ is

$$
{ }^{q} \mathcal{L}_{\xi} V_{i}=\bigoplus_{x, k}^{q}\left(V_{i}\right) \xi^{k}+\bigoplus_{x, i}^{q}\left(\xi^{k}\right) V_{k}
$$

The simplest case, a scalar function, has fractional Lie derivative

$$
{ }^{q} \mathcal{L}_{\xi} f=\xi^{k} \bigoplus_{x, k}^{q}(f) .
$$

Some properties of the fractional Lie derivative are discussed in the Appendix.

\section{A FRACTIONAL THIN LAYER}

\section{A. Describing the layer}

Many of the thin shell examples considered in the literature ${ }^{22-34}$ are descriptions of a boundary layer between two space times, $M^{ \pm}$. Boundary layers are often treated in the Israel formalism ${ }^{35}$ which models a thin shell as a layer of zero thickness. The basic input into this description is the extrinsic curvature of the boundary layer as seen by the bounding space times.

$$
K_{c d}^{ \pm}=\frac{1}{2} \mathcal{L}_{N}\left(g_{i j}^{ \pm}\right) h_{c}^{ \pm i} h_{d}^{ \pm j}
$$

where $c, d$ range over the coordinates of the boundary layer, $N^{i}$ is the normal to the layer, and $h_{i j}$ is the projection operator onto the layer,

$$
h_{i j}^{ \pm}=g_{i j}^{ \pm}-N_{i}^{ \pm} N_{j}^{ \pm} .
$$

The jump in the extrinsic curvatures, $\left\langle K_{a b}\right\rangle=K_{c d}^{+}-K_{c d}^{-}$, and their trace $\langle K\rangle=K^{+}-K^{-}$are related to the stress energy of the single boundary layer. ${ }^{35}$

$$
-8 \pi S_{a b}=\left\langle K_{a b}\right\rangle-\langle K\rangle h_{a b} .
$$

The fractional extrinsic curvature is defined in terms of the fractional Lie derivative.

$$
{ }^{q} K_{c d}=\frac{1}{2}{ }^{q} \mathcal{L}_{N}\left(g_{i j}\right) h_{c}^{i} h_{d}^{j},
$$

where

$$
{ }^{q} \mathcal{L}_{N}\left(g_{i j}\right)=N^{k} \bigoplus_{x, k}^{q}\left(g_{i j}\right)+g_{k j} \bigoplus_{x, i}^{q}\left(N^{k}\right)+g_{i k} \bigoplus_{x, j}^{q}\left(N^{k}\right)
$$

and Eq. (7) relates $\bigoplus_{x, k}^{q}$ to the fractional derivative form. The fractional stress energy is defined through jumps in the fractional extrinsic curvature,

$$
-8 \pi^{q} S_{a b}=\left\langle{ }^{q} K_{a b}\right\rangle-\left\langle{ }^{q} K\right\rangle h_{a b} .
$$




\section{B. Using the fractional extrinsic curvature}

The fractional extrinsic curvature is defined as an integral over a shell of thickness $\Delta$. Because of this nonlocal integral structure, one might assume that the usual Israel jump in extrinsic curvature could be replaced by a single extrinsic curvature calculation. However, just as for the regular Lie derivative, the fractional Lie derivative, while it includes a thickness component, only provides the value of the derivative at a single space-time point. For example, consider a shell bounded by two Minkowski space times. We would expect no jump discontinuities across such a boundary.

$$
M^{ \pm}: d s^{2}=-d t^{2}+d r^{2}+r^{2} d \Omega^{2}
$$

The unit normal to the shell is $N^{i}=(0,1,0,0) .{ }^{q} K_{\theta \theta}$ and ${ }^{q} K_{\phi \phi}$ will be nonzero for each of Minkowski boundaries. Using Eqs. (7), (26), and (27), with $\eta=r$, the fractional extrinsic curvature is

$$
{ }^{q} K_{c d}=\frac{\Gamma(2-q)}{2(\Delta))^{1-q}} N^{r} D_{r-\Delta, r}^{q}\left(g_{i j}\right) h_{c}^{i} h_{d}^{j}
$$

For Minkowski we have

$$
\begin{gathered}
{ }^{q} K_{\theta \theta}^{M}=\frac{\Gamma(2-q)}{2 \Gamma(1-q)(\Delta)^{1-q}} \int_{r-\Delta}^{r} d y 2 y(r-y)^{-q}, \\
{ }^{q} K_{\theta \theta}^{M}=\frac{\Gamma(2-q) 2 R_{o}^{2-q}}{2 \Gamma(1-q)(\Delta)^{1-q}} B_{\Delta / R_{o}}(1-q, 2),
\end{gathered}
$$

where $B_{\Delta / R}(1-q, 2)$ is the incomplete beta function and $r$ was set equal to the boundary value, $R_{o}$.

$$
B_{x}(a, b)=\int_{0}^{x} t^{a-1}(1-t)^{b-1} .
$$

Using the beta function expansion ${ }^{36}$

$$
B_{x}(a, b)=x^{a}\left(\frac{1}{a}+\frac{1-b}{a+1} x+\frac{(1-b)(2-b)}{2 !(a+2)} x^{2}+\cdots\right),
$$

we have

$$
\begin{gathered}
{ }^{q} K_{\theta \theta}^{M}=\frac{\Gamma(2-q) 2 R_{o}^{2-q}}{2 \Gamma(1-q)(\Delta)^{1-q}}\left(\frac{\Delta}{R_{o}}\right)^{1-q}\left(\frac{1}{1-q}-\frac{1}{2-q} \frac{\Delta}{R_{o}}+\cdots\right), \\
{ }^{q} K_{\theta \theta}^{M} \sim R_{o}\left(1-\frac{1-q}{2-q} \frac{\Delta}{R_{o}}+\cdots\right) .
\end{gathered}
$$

Because of the integral over the shell, this single extrinsic curvature might be regarded as the entire jump contribution. However, if there were no boundary layer between the two space times, one would expect the jump in the extrinsic curvatures to be zero. Instead, the fractional extrinsic curvature, in the $\Delta->0$ limit, is simply the regular extrinsic curvature evaluated over the outer bounding surface. The expression above is only the extrinsic curvature evaluated with one of the bounding space times. In order to use the fractional extrinsic curvature in an Israel layer calculation, it has to be calculated for both interior and exterior boundaries, just as in the nonfractional case. In this example, there is no jump in the extrinsic curvatures across the boundary as expected. 


\section{TWO EXAMPLES}

In this section two examples of fractional shells are considered. One layer has cylindrical symmetry and is bounded by Levi-Civita and Minkowski. The second layer has spherical symmetry and separates Schwarzschild and Minkowski. Dynamic shells have broad applications as reflected by the large literature dealing with their evolution. ${ }^{22-34}$ The two examples considered in this section are static and are presented as examples of the use of the fractional Lie derivative in the Israel formalism. Dynamic fractional shells will be considered elsewhere.

\section{A. A layer between Minkowski and Levi-Civita}

A simple cylindrical example is a layer separating an interior Minkowski and a vacuum Levi-Civita with angular deficit factor $\delta$,

$$
\begin{gathered}
M^{+}: d s^{2}=-d t^{2}+d r^{2}+\delta^{2} r^{2} d \phi^{2}+d z^{2}, \\
M^{-}: d s^{2}=-d t^{2}+d \rho^{2}+\rho^{2} d \phi^{2}+d z^{2} .
\end{gathered}
$$

Using the result from the previous section, Eq. (33), the nonzero fractional extrinsic curvatures are ${ }^{q} K_{\theta \theta}$,

$$
\begin{gathered}
{ }^{q} K_{\theta}^{\theta M-} \simeq R_{o}\left(1-\frac{1-q}{2-q} \frac{\Delta}{R_{o}}+\cdots\right), \\
{ }^{q} K_{\theta}^{\theta M+} \simeq \delta R_{o}\left(1-\frac{1-q}{2-q} \frac{\Delta}{R_{o}}+\cdots\right) .
\end{gathered}
$$

Calculating the jumps we find

$$
\begin{aligned}
\left\langle{ }^{q} K\right\rangle \simeq & R_{o}(\delta-1)\left(1-\frac{1-q}{2-q} \frac{\Delta}{R_{o}}+\cdots\right), \\
\left\langle{ }^{q} K_{\theta}^{\theta}\right\rangle \simeq & R_{o}(\delta-1)\left(1-\frac{1-q}{2-q} \frac{\Delta}{R_{o}}+\cdots\right), \\
& -8 \pi S_{j}^{i}=\left\langle{ }^{q} K_{j}^{i}\right\rangle-\left\langle{ }^{q} K\right\rangle h_{j}^{i}, \\
8 \pi S_{t}^{t} \simeq & -R_{o}(1-\delta)\left(1-\frac{1-q}{2-q} \frac{\Delta}{R_{o}}+\cdots\right) .
\end{aligned}
$$

The stress energy in the layer has positive density and no pressure. In a perfect fluid model this could be dust. The method of including a fractional parameter in the regular Israel layer ${ }^{17,18}$ by matching fractional derivatives across the boundary would require no angular deficit, $\delta=1$, for these two space times. In this example, the stress energy of the layer only includes a thin layer contribution for $q \neq 1$, the fractional case. The $\Delta$ dependence was included by using the nonlocality of the fractional derivative. In the $q->1$ limit, the fractional derivative becomes a regular local derivative and the effect vanishes.

\section{B. A layer between Minkowski and Schwarzschild}

\section{The fractional extrinsic curvature}

Consider a shell bounded by the an exterior Schwarzschild space time and an interior Minkowski at $r=R_{o}$, 


$$
\begin{gathered}
M^{+}: d s^{2}=-\left(1-\frac{2 m}{r}\right) d t^{2}+\frac{d r^{2}}{1-(2 m / r)}+r^{2} d \Omega^{2}, \\
M^{-}: d s^{2}=-\left(1-\frac{2 m}{R_{o}}\right) d t^{2}+d r^{2}+r^{2} d \Omega^{2} .
\end{gathered}
$$

For this calculation the normal vectors have only radial components and we have

$$
{ }^{q} \mathcal{L}_{N}\left(g_{i j}\right)=N^{r} \bigoplus_{r-\Delta, r}^{q}\left(g_{i j}\right)+g_{r j} \bigoplus_{r-\Delta, i}^{q}\left(N^{r}\right)+g_{i r} \bigoplus_{r-\Delta, j}^{q}\left(N^{r}\right)
$$

with

$$
\bigoplus_{r-\Delta, r}^{q}\left(g_{i j}\right)=\frac{\Gamma(2-q)}{\Gamma(1-q)(\Delta)^{1-q}} \int_{r-\Delta}^{r} \frac{\partial g_{i j}(w)}{\partial w}(r-w)^{-q} d w
$$

and $q \leqslant 1, r->R_{o}$ on the boundary. For the interior Minkowski space time, $N^{i}=(0,1,0,0)$ and only ${ }^{q} K_{\theta \theta}$ and ${ }^{q} K_{\phi \phi}$ will contribute and the result has been calculated above. From Eq. (33) we have

$$
{ }^{q} K_{\theta}^{\theta}={ }^{q} K_{\phi}^{\phi}=\frac{1}{R_{o}}\left(1-\frac{1-q}{2-q} \frac{\Delta}{R_{o}}+\cdots\right) .
$$

For the exterior Schwarzschild (SC) space time, $N^{i}=(0, \sqrt{1-(2 m / r)}, 0,0)$ and ${ }^{q} K_{\theta \theta},{ }^{q} K_{\phi \phi}$, and ${ }^{q} K_{t t}$ will contribute. Except for the structure of the normal, the angular extrinsic curvatures will be the same as in Minkowski and we have at the boundary

$$
{ }^{q} K_{\theta}^{\theta}={ }^{q} K_{\phi}^{\phi}=\frac{\sqrt{1-\left(2 m / R_{o}\right)}}{R_{o}}\left(1-\frac{1-q}{2-q} \frac{\Delta}{R_{o}}+\cdots\right) .
$$

Calculating ${ }^{q} K_{t t}$ and letting $r->R_{o}$ we have

$$
\begin{gathered}
{ }^{q} K_{t t}=\frac{N^{r}}{2} \bigoplus_{r-\Delta, r}^{q}\left(g_{t t}\right)=2 m \frac{\sqrt{1-\left(2 m / R_{o}\right)}}{2} \bigoplus_{r-\Delta, r}^{q}\left(r^{-1}\right), \\
{ }^{q} K_{t t}=-2 m \frac{\sqrt{1-\left(2 m / R_{o}\right)}}{2} \frac{\Gamma(2-q) R_{o}^{-1-q}}{\Gamma(1-q) \Delta^{1-q}} B_{\Delta / R_{o}}(1-q,-1) .
\end{gathered}
$$

The negative one beta function parameter is permitted for $\Delta / R_{o} \neq 1 .^{36}$ This expression is most easily expanded in terms of the hypergeometric function ${ }_{2} F_{1}(a, b, c ; x)$,

$$
B_{\Delta / R_{o}}(1-q,-1)=\frac{\left(\Delta / R_{0}\right)^{1-q}}{1-q}{ }_{2} F_{1}\left(1-q, 2,2-q ; \frac{\Delta}{R_{o}}\right)=\frac{\left(\Delta / R_{o}\right)^{1-q}}{1-q}\left(1+\frac{1-q}{2-q} 2 \frac{\Delta}{R_{o}}+\cdots\right)
$$

and we have

$$
{ }^{q} K_{t}^{t}=\frac{m}{\sqrt{1-\left(2 m / R_{o}\right)} R_{o}^{2}}\left(1+\frac{1-q}{2-q} 2 \frac{\Delta}{R_{o}}+\cdots\right) .
$$




\section{The layer stress energy}

Using the ${ }^{q} K_{a b}$ calculated in the previous section, the jumps are

$$
\begin{gathered}
\left\langle{ }^{q} K_{\theta}^{\theta}\right\rangle=\left\langle{ }^{q} K_{\phi}^{\phi}\right\rangle=\frac{\left(\sqrt{1-\left(2 m / R_{o}\right)}-1\right)}{R_{o}}\left(1-\frac{1-q}{2-q} \frac{\Delta}{R_{o}}+\cdots\right) \\
\left\langle{ }^{q} K_{t}^{t}\right\rangle=\frac{m}{\sqrt{1-\left(2 m / R_{o}\right)} R_{o}^{2}}\left(1+2 \frac{1-q}{2-q} \frac{\Delta}{R_{o}}+\cdots\right) .
\end{gathered}
$$

With these jumps, the layer energy density, $\sigma$, and stress, $P$, are

$$
\begin{gathered}
8 \pi S_{t}^{t}=-8 \pi \sigma=-\left\langle{ }^{q} K_{\theta}^{\theta}+{ }^{q} K_{\phi}^{\phi}\right\rangle, \\
8 \pi S_{\theta}^{\theta}=8 \pi P=\left\langle{ }^{q} K_{t}^{t}+{ }^{q} K_{\phi}^{\phi}\right\rangle .
\end{gathered}
$$

Substituting we have

$$
\begin{gathered}
8 \pi \sigma=2 \frac{\left(1-\sqrt{1-\left(2 m / R_{o}\right)}\right)}{R_{o}}\left(1-\frac{1-q}{2-q} \frac{\Delta}{R_{o}}+\cdots\right), \\
8 \pi P=\frac{1}{R_{o} \sqrt{1-\left(2 m / R_{o}\right)}}\left\{+1-\frac{m}{R_{o}}-\sqrt{1-\frac{2 m}{R_{o}}}+\frac{1-q}{2-q} \frac{\Delta}{R_{o}}\left(\frac{4 m}{R_{o}}-1+\sqrt{1-\frac{2 m}{R_{o}}}\right) \ldots\right\} .
\end{gathered}
$$

In the $m \ll R_{o}$ limit the stress energy becomes

$$
\begin{gathered}
\sigma \approx \frac{m}{4 \pi R_{o}^{2}}\left(1-\frac{1-q}{2-q} \frac{\Delta}{R_{o}}\right), \\
P \approx \frac{3 m}{8 \pi R_{o}^{2}} \frac{1-q}{2-q} \frac{1}{R_{o}} .
\end{gathered}
$$

For $q=1$, the density is simply the SC mass parameter over the surface area of the sphere and the fluid is dust. For $q<1$ the reduction in density, for the same mass, means the same mass is distributed over a larger region. Since the size of the sphere is set, the reduction in the areal density is possible if the mass is being distributed in an annular volume rather than totally over an area. In addition, the appearance of the fractional correction factor suggests that the thickness of the shell is parametrized by $q$. One could define a parametrized shell thickness,

$$
\Delta_{q}=\frac{1-q}{2-q} \Delta .
$$

With this parametrization, a family of shells is created with the thickest shells occurring for $q$ close to zero.

\section{DISCUSSION}

The Israel formalism models a thin shell of matter as a zero thickness layer. The stress energy of the layer is related to jumps in the extrinsic curvature across the single boundary. In this paper, a fractional Lie derivative was developed and used to calculate a fractional extrinsic curvature which was input into the Israel formalism. The layer being described still has zero thickness; a single coordinate boundary is used to calculate the curvature jumps, but the nonlocal fractional derivative used to define the fractional extrinsic curvature allows the inclusion of a contribution 
from a thin layer thickness as well as a fractional parameter, $q$, in the layer stress energy. The fractional parameter can be used to parametrize the shell thickness, creating a family of shells of varying thickness. The development is for $q \leqslant 1$ but can be extended to higher values of the fractional parameter. As an example of the use of the fractional Lie derivative within the Israel formalism, the method was applied to a cylindrical layer between a Minkowski interior and a Levi-Civita exterior and a spherical Schwarschild-Minkowski shell. The inclusion of the shell thickness is a fractional effect and vanishes as the fractional parameter approaches one.

There are choices to be made in the development of fractional formalisms applicable in general relativity. There are many fractional derivative definitions, Caputo, Riesz, RiemannLiouville, etc., and the choice of derivative will depend on the application. The layers discussed here involve one-dimensional fractional Lie transport in a spatial variable but multidimensional transport processes will be important in the dynamics of fractional layers. Fractional derivatives have successfully described random walk processes that are heavy tailed in either jump size or jump timing and this has provided avenues for higher dimensional generalizations of the fractional derivative. Umarov and Gorenflo ${ }^{37}$ have discussed a multispace dimension random walk model that is related to diffusion that is fractional in its spatial derivatives. Gorenflo and Mainardi have presented some random walk models discrete in both space and time. ${ }^{38}$ Meerschaert and co-workers, ${ }^{39,40}$ have begun to develop a fractional derivative operator that may be used when space and time variables are linked. Generalizing the formalism to describe the dynamic fractional layers with plane and spherical symmetries in general relativity ${ }^{41-43}$ would be both useful and interesting.

\section{APPENDIX: FRACTIONAL DETAILS}

\section{The Caputo derivative}

The Caputo derivative of a scalar function can generally be written as ${ }^{4}$

$$
D_{x}^{q} f\left(x^{\prime}\right)=\frac{1}{\Gamma(m-q)} \int_{x}^{x^{\prime}} f^{(m)}(z)\left(x^{\prime}-z\right)^{m-1-q} d z
$$

where $m-1<q \leqslant m, m$ integer. $f^{(m)}=d^{m} f / d z^{m}$. The development in the text was for $m=1$. The fractional derivative is an interpolator between regular integer derivatives. To move to $q>1$ would require $m=2$ and we would have

$$
D_{x}^{q} f\left(x^{\prime}\right)=\frac{1}{\Gamma(2-q)} \int_{x}^{x^{\prime}} \frac{d^{2} f(z)}{d z^{2}}\left(x^{\prime}-z\right)^{1-q} d z .
$$

Integrating by parts we have

$$
D_{x}^{q} f\left(x^{\prime}\right)=-\left(\frac{d f}{d z}\right)_{x}\left(x^{\prime}-x\right)^{1-q}+\frac{1}{\Gamma(1-q)} \int_{x}^{x^{\prime}} \frac{d f(x)}{d z}\left(x^{\prime}-z\right)^{-q} d z .
$$

Remembering that for $m=2, q>1$, one can see the interpolating term.

\section{Notation}

This section contains a list of the notations used. The starting point is the Caputo derivative discussed in the previous section, $D_{x}^{q}()$ with an additional index, $k$, to define the coordinate partial occurring in the definition.

$$
D_{x, k}^{q} V^{i}\left(x^{\prime}\right)=\frac{\partial^{q} x^{\prime i}}{\partial\left(x^{\prime k}-x^{k}\right)^{q}}=\frac{1}{\Gamma(1-q)} \int_{x}^{x^{\prime}} d y \frac{\partial V^{i}(y)}{\partial y^{k}}\left(x^{\prime}-y\right)^{-q} .
$$

The integral is over the thickness of the layer with $x=\eta-\Delta, x^{\prime}=\eta$. The $\bigoplus_{x}^{q}$ derivative is defined in terms of the Caputo derivative, 


$$
D_{x, k}^{q}()=\frac{\left(x^{\prime}-x\right)^{1-q}}{\Gamma(2-q)} \bigoplus_{x, k}^{q}() .
$$

An example of its use are found in the next section. The fractional Lie derivative is defined in terms of $\bigoplus_{x, k}^{q}()$,

$$
\begin{gathered}
{ }^{q} \mathcal{L}_{\xi} V^{i}=\bigoplus_{x, k}^{q}\left(V^{i}\right) \xi^{k}-\bigoplus_{x, k}^{q}\left(\xi^{i}\right) V^{k}, \\
{ }^{q} \mathcal{L}_{\xi} V_{i}=\bigoplus_{x, k}^{q}\left(V_{i}\right)\left(\xi^{k}\right)+V_{k} \bigoplus_{x, i}^{q}\left(\xi^{k}\right), \\
{ }^{q} \mathcal{L}_{\xi \xi} f=\xi^{k} \bigoplus_{x, k}^{q}(f) .
\end{gathered}
$$

The fractional extrinsic curvature is defined in terms of the fractional Lie derivative generated by the normal to the layer. $g_{i j}$ is the metric of the bounding space time and $h_{i j}$ is the projection operator onto the layer,

$$
{ }^{q} K_{c d}=\frac{1}{2}{ }^{q} \mathcal{L}_{N}\left(g_{i j}\right) h_{c}^{i} h_{d}^{j} .
$$

Jumps across the layer are described by

$$
\left\langle{ }^{q} K_{c d}\right\rangle
$$

\section{The $\oplus^{q}$ derivative}

The $\bigoplus^{q}$ derivative defined in Eq. (7) in the text as a replacement for the regular partial derivative divides out some of the usual fractional derivative behavior. Consider the Caputo derivative of $r^{2}$ over a spherical region from the origin to $r$. We have

$$
D_{r}^{q} r^{2}=\frac{1}{\Gamma(1-q)} \int_{0}^{r} 2 z(r-z)^{-q} d z=\frac{2 r^{2-q}}{\Gamma(1-q)} \int_{0}^{1} w(1-w)^{-q} d w=\frac{2 r^{2-q}}{\Gamma(1-q)} \frac{\Gamma(1-q) \Gamma(2)}{\Gamma(2-q)}=\frac{2 r^{2-q}}{\Gamma(3-q)} .
$$

The $\bigoplus^{q}$ derivative is

$$
\begin{gathered}
D_{x}^{q}()=\frac{\left(x^{\prime}-x\right)^{1-q}}{\Gamma(2-q)} \bigoplus_{x}^{q}(), \\
\bigoplus^{q} r^{2}=\frac{2 r}{2-q} .
\end{gathered}
$$

\section{Some properties of the fractional Lie derivative}

\section{a. Defining the fractional commutator}

The regular Lie derivative defines the commutator as

$$
\mathcal{L}_{U} V=[U, V]=\nabla_{U} V-\nabla_{V} U,
$$

where $\nabla_{U}=U^{k} \nabla_{k}$ is the standard directional derivative. The fractional Lie derivative generalizes the commutator,

$$
\begin{gathered}
{ }^{q} \mathcal{L}_{U} V^{i}=U^{k} \bigoplus_{x, k}^{q} V^{i}-V^{k} \bigoplus_{x, k}^{q} U, \\
{ }^{q}[U, V]^{i}=U^{k} \bigoplus_{x, k}^{q} V^{i}-V^{k} \bigoplus_{x, k}^{q} U^{i} .
\end{gathered}
$$




\section{Product of derivatives}

The product of two regular Lie derivatives is related to the Lie derivative of the commutator.

$$
\mathcal{L}_{[U, V]}=\mathcal{L}_{U} \mathcal{L}_{V}-\mathcal{L}_{V} \mathcal{L}_{U}
$$

Consider the fractional Lie derivative acting on a scalar function $f$,

$$
{ }^{q} \mathcal{L}_{[U, V]} f=\left\{U^{k} \bigoplus_{x, k}^{q} V^{i}-V^{k} \bigoplus_{x, k}^{q} U^{i}\right\} \bigoplus_{x, i}^{q} f=U^{k} \bigoplus_{x, k}^{q} V^{i} \bigoplus_{x, i}^{q} f-V^{k} \bigoplus_{x, k}^{q} U^{i} \bigoplus_{x, i}^{q} f={ }^{q} \mathcal{L}_{U}^{q} \mathcal{L}_{V} f-{ }^{q} \mathcal{L}_{V}{ }^{q} \mathcal{L}_{U} f
$$

and the fractional derivative has the same structure.

${ }^{1}$ K. B. Oldham and J. Spanier, The Fractional Calculus (Academic, New York, 1974), Chap. 1.

${ }^{2}$ S. G. Samko, A. A. Kilbas, and O. I. Marichev, Fractional Integrals and Derivatives: Theory and Applications (Gordon and Breach Science, New York, 1993).

${ }^{3}$ G. M. Zaslavsky, Phys. Rep. 371, 461 (2002).

${ }^{4}$ R. Gorenflo and F. Mainardi, in Fractals and Fractional Calculus in Continuum Mechanics, edited by A. Carpinteri and F. Mainardi (Springer, New York, 1997).

${ }^{5}$ R. Gorenflo, G. De Fabritiis, and F. Mainardi, Physica A 269, 79 (1999).

${ }^{6}$ A. I. Saichev and G. M. Zaslavsky, Chaos 7, 753 (1997).

${ }^{7}$ W. R. Schneider and W. Wyss, J. Math. Phys. 30, 34 (1989).

${ }^{8}$ E. Barkai, Phys. Rev. E 63, 046118 (2001).

${ }^{9}$ E. Scalas, R. Gorenflo, F. Mainardi, and M. Raberto, in Scaling and Disordered Systems, edited by F. Family et al. (World Scientific, Singapore, 2002), pp. 281-289.

${ }^{10}$ N. Laskin, Phys. Rev. E 66, 056108 (2002).

${ }^{11}$ Mark Naber, J. Math. Phys. 45, 3339 (2004).

${ }^{12}$ Applications of Fractional Calculus in Physics, edited by R. Hilfer (World Scientific, Singapore, 2000).

${ }^{13}$ I. Podlubny, Fractional Differential Equations (Academic, New York, 1999), p. 86.

${ }^{14}$ K. S. Miller and B. Ross, An Introduction to the Fractional Calculus and Fractional Differential Equations (Wiley, New York, 1993).

${ }^{15}$ F. Riewe, Phys. Rev. E 53, 1890 (1996); 55, 3581 (1997).

${ }^{16}$ D. W. Dreisigmeyer and P. M. Young, J. Phys. A 36, 8297 (2003).

${ }^{17}$ J. P. Krisch, J. Math. Phys. 46, 042506 (2005).

${ }^{18}$ S. Bayin, E. N. Glass, and J. P. Krisch, J. Math. Phys. 47, 012501 (2006).

${ }^{19}$ J. Munkhammar, Rose-Hulman Institute of Technology Undergraduate Math Journal (2005), www.rose-hulman.edu/ mathjournal/.

${ }^{20}$ K. M. Kolwankar and A. Gangal, Chaos 6, 505 (1996).

${ }^{21}$ K. Cotrill-Shepherd and M. Naber, J. Math. Phys. 42, 2203 (2001).

${ }^{22}$ V. A. Berezin, V. A. Kuzmin, and I. I. Tkachev, Phys. Lett. 120B, 91 (1983).

${ }^{23}$ K. Maeda, Gen. Relativ. Gravit. 18, 931 (1986).

${ }^{24}$ V. A. Berezin, V. A. Kuzmin, and I. I. Tkachev, Phys. Rev. D 36, 2919 (1987).

${ }^{25}$ J. Ipser, Phys. Rev. D 36, 1933 (1987).

${ }^{26}$ D. Garfinkle and C. Vuille, Class. Quantum Grav. 6, 1819 (1989).

${ }^{27}$ N. Sakai and K.-I. Maeda, Phys. Rev. D 50, 5425 (1994).

${ }^{28}$ S. Ansoldi, A. Aurilia, Y. Balbinsot, and E. Spallucci, Class. Quantum Grav. 14, 2727 (1997).

${ }^{29}$ U. Kirchner, Class. Quantum Grav. 21, 3845 (2004).

${ }^{30}$ S. Khosravi, S. Khakshournia, and R. Mansouri, Class. Quantum Grav. 23, 5927 (2006).

${ }^{31}$ J. Bicak and B. G. Schmidt, Astrophys. J. 521, 708 (1999).

${ }^{32}$ A. Wang and P. S. Letelier, Phys. Rev. D 51, R6612 (1995).

${ }^{33}$ S. K. Blau, E. I. Guendelman, and A. H. Guth, Phys. Rev. D 35, 1747 (1987).

${ }^{34}$ W. Israel, Nuovo Cimento B 44, 1 (1966); 48, 463 (1967).

${ }^{35}$ E. Poisson, A Relativist's Toolkit (Cambridge University Press, Cambridge, 2004).

${ }^{36}$ G. B. Arfken and H. J. Weber, Mathematical Methods for Physicists (Academic, New York, 1995), p. 301.

${ }^{37}$ S. Umarov and R. Gorenflo, Fractional Calculus Appl. Anal. 8, 73 (2005).

${ }^{38}$ R. Gorenflo and F. Mainardi, Problems and Methods in Mathematical Physics: Proceedings of the 11th TMP (Chemnitz, Germany), edited by J. Elschner, I. Gohberg, and B. Silvermann (Birkhauser Verlag, Basel-Boston-Berlin, 2001), p. 120.

${ }^{39}$ B. Baeumer, M. M. Meerschaert, and J. Mortensen, Proc. Am. Math. Soc. 133, 2273 (2005).

${ }^{40}$ M. M. Meerschaert, D. A. Benson, H. P. Scheffler, and B. Baeumer, Phys. Rev. E 65, 1103 (2002).

${ }^{41}$ S. Chassemi, S. Khakshournia, and R. Mansouri, e-print gr-qc/0505073.

${ }^{42}$ M. Khorrami and R. Mansouri, Phys. Rev. D 44, 557 (1991).

${ }^{43}$ S. Khakhshournia and R. Mansouri, Gen. Relativ. Gravit. 34, 1847 (2002). 